\title{
Performance analysis of energy-harvesting-aware multi-relay networks in Nakagami-m fading
}

\author{
Shaohong Zhong ${ }^{1,2}$, Huajun Huang ${ }^{2}$ and Renfa Li ${ }^{\text {* }}$
}

\begin{abstract}
This paper investigates the outage performance of an energy-harvesting(EH) relay-aided cooperative network, where the source node transmits information to its destination node with the help of multiple energy-harvesting cooperative nodes. For such a system, we derive an explicit closed-form expression of outage probability over Nakagami-m fading channels, where the on-off EH model is considered. We simulate the explicit closed-form expression of outage probability with different parameters compared with the related Monte Carlo method. It is shown that the simulation results match well with the numerical ones, which demonstrates the correctness of our theoretical analysis. It indicates that to evaluate the system outage performance, our theoretical results can be applied without running the complex Monte Carlo simulation. Via simulations, it is observed that the system outage performance will be improved when natural source EH relays are employed. Besides, more relays, better system performance.
\end{abstract}

Keywords: Energy harvesting, Nakagami-m fading channel, Outage probability, Relay-aided cooperative networks

\section{Introduction}

Energy-constrained wireless networks, such as sensor networks, are typically powered by batteries, which have limited operation time. Although replacing and recharging the batteries artificially can prolong the lifetime of the network, it is with high cost in practice, especially in large-scale networks with hundreds or thousands nodes. Besides in a hazardous environment (e.g., in toxic environments) or for sensors embedded in building structures or inside human bodies, it is also impossible to replace the batteries periodically.

Recently, energy harvesting, which can collect energy (such as solar energy, wind energy, and even radiofrequency signal) from renewable resource in the ambient environment, has become a promising solution to prolong the lifetime of energy-constrained wireless networks [1-6]. It is considered as an appealing approach to power wireless node, especially low-power devices.

\footnotetext{
*Correspondence: rfli@vip.sina.cn

${ }^{1}$ College of Comuputer Science and Electronic Engineering, and the Key

Laboratory for Embedded and Network Computing, Hunan University,

Changsha, People's Republic of China

Full list of author information is available at the end of the article
}

Relay-aided wireless networks exploit special diversity to improve system performance [7-9]. To exploit these networks' advantages, relay nodes harvesting energy from ambient environments have been investigated. For example, in [10], the authors designed a wireless communication device relying exclusively on energy harvesting. The work of [11] focused on the performance analysis of an energy-harvesting relay-aided cooperative network under slow fading channel from a perspective of outage behavior and deriving the closed-form expression of outage probability of the proposed cooperative protocol. In [12], an amplify-and-forward (AF) relaying network was studied, where an energy-constrained relay node harvests energy from the received RF signal and uses that harvested energy to forward the source information to the destination. Based on the time switching and power splitting receiver architectures, two relaying protocols were proposed to enable energy harvesting and information processing at the relay $[13,14]$.

Outage probability, as one of the most commonly used performance metric in wireless cooperative system over the fading channel, has attracted considerable research interests in the past decades [15, 16]. In [17], an opportunistic relaying with the AF strategy was presented and

\section{Springer Open}

(c) The Author(s). 2018 Open Access This article is distributed under the terms of the Creative Commons Attribution 4.0 International License (http://creativecommons.org/licenses/by/4.0/), which permits unrestricted use, distribution, and reproduction in any medium, provided you give appropriate credit to the original author(s) and the source, provide a link to the Creative Commons license, and indicate if changes were made. 
the outage probability was discussed under Rayleigh fading channels. In [18], outage probability of dual-hop decode-and-forward (DF) relaying scheme was analyzed over mixed Rayleigh and generalized Gamma fading channels. In [19], the authors analyzed the outage probability of opportunistic AF orthogonal frequency-division multiplexing (OFDM) relaying over Nakagami-m fading channel and presented a closed-form outage probability of the proposed system at high signal-to-noise (SNR) regime. In [20], the paper analyzed the performance of two-way amplify-and-forward relaying over Nakagami-m frequency-selective fading channels taking consideration of the multipath spread of the channel. In [21], the authors presented a general two-parameter received SNR model for two-hop AF relaying.

Although lots of important results have been presented in the above existing works, there still some fundamental issues to be studied for multi-relay EH systems $[22,23]$. For example, the works of [11] and [12] focused on the research of outage probability with harvesting energy relay-aided cooperative networks on the Rayleigh channel, but did not explore it over the Nakagami-m fading channnel. The work of [17] and [18] focused on the outage probability of relay-aided networks over Rayleigh channel with AF and DF schemes, respectively, but they did not consider the energy harvesting from an ambient environment. The works of $[19,20]$, and [21] analyzed the performance of two-hop AF relaying over Nakagami-m fading channels, but did not consider on energy harvesting from an ambient environment.

In this paper, we consider a relay-aided cooperative wireless network, where relay nodes are able to harvest energy from ambient environment. An on-off EH model is assumed and Nakagami-m fading channels is assumed, as it is a more general channel model, where the Rayleigh model can be consider as a special case of it. Our aim is to study the outage probability.

Our contributions are as follows. We analyze the outage performance of an energy-harvesting relay-aided cooperative network in Nakagami-m fading channel and derive an explicit closed-form expression of outage probability for the on-off relay-aided cooperative protocol with the help of the Markov property of energy buffer status. Monte Carlo simulations demonstrate the correctness of our derived outage probability express. It is also shown that the outage performance can be significantly improved especially in high SNR regime, and the more the workable relay-aided nodes take part in relay aiding, the better system performance can be got.

The rest of the paper is organized as follows. Section 2 reports about model of the system and the on-off energyharvesting model. Section 3 presents the performance analysis of outage probability. Section 4 presents the numerical results and validates the analytical results through Monte Carlo simulations. Finally, section 5 concludes the paper.

\section{System model}

\subsection{Network model}

Consider an energy-harvesting relay-aided systems where the source node $S$, communicates with its destination node $D$ with the help of energy-harvesting relay-aided nodes $R_{k}(1 \leq \kappa \leq \mathrm{K})$, where the direct link between $S$ and $D$ exists in the system as illustrated in Fig. 1.

The baseband-equivalent discrete time mode for the channels $S-D$ is assumed; therefore, the signal received over the direct link can be expressed by

$$
y_{s, d}[n]=\sqrt{P_{s}} h_{s, d}[n] x_{s}[n]+n_{d}[n], n=1,2, \ldots, N
$$

where $N$ denotes the whole signal block length, and $y_{s, d}[n]$ denotes the received signal at $D . h_{s, d}[n]$ and $n_{d}[n]$ denotes the channel fading coefficient and additive noise, respectively. $P_{S}$ denotes source node $S$ 's transmission power.

For the relay node, AF relay operation is employed. Provided that there is harvesting energy used for transmitting current signal block at $R_{k}$, information will be transmitted from $S$ to $R_{k}$ first and then to $D$ from $R_{k}$. In order to avoid interference between links $S-R_{k}$ and $R_{k}-D$, half-duplex transmission is assumed, and each signal block is divided into two subphases. In the first subphase, information is transmitted from $S$ to $R_{k}$, which is given by

$y_{s, r_{k}}[n]=\sqrt{P_{s}} h_{s, r_{k}}[n] x_{s}[n]+n_{r_{k}}[n], n=1,2, \ldots, N / 2$

where $y_{s, r_{k}}[n], n_{r_{k}}[n]$, and $h_{s, r_{k}}[n]$ denotes the received signal, additive noise at $R_{k}$, and the fading coefficient between $S$ and $R_{k}$ respectively. Then, the received information is forwarded by $R_{k}$ to $D$ in the second subphase, which is expressed by

$y_{r_{k}, d}[n]=\sqrt{P_{r}} h_{r_{k}, d}[n] x_{r_{k}}[n]+n_{d}[n], n=N / 2+1, \ldots, N$

where $x_{r_{k}}[n], P_{r}$, and $h_{r_{k}, d}[n]$ denotes the transmitted signal, the transmit power at $R_{k}$, and the channel fading coefficient between $R_{k}$ and $D$ respectively.

\subsection{On-off energy-harvesting model}

At first, we consider the situation that the relay $R_{k}$ does not have an energy storage device; it can only work at the current block with the energy $E_{i}$, and we assume that $E_{r}$ is the minimal energy, that is, each relay node activate itself and forward signal. If the energy $E_{i}$ is less than $E_{r}$, transmission outage will occur.

Secondly, we consider the situation where the relay $R_{k}$ has energy storage with capacity $E_{\max }$, and $S_{i}$ denote the 


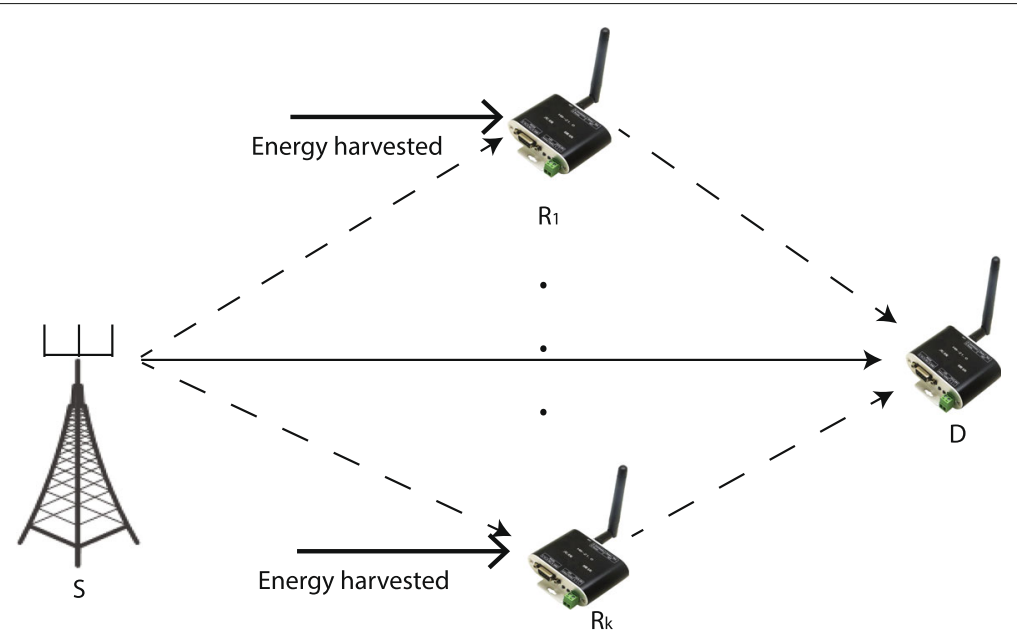

Fig. 1 A system structure for energy-harvesting relay-aided cooperative network with $K$ available relay nodes, and the information flow is intended to be transmitted from $S$ to $D$, where the solid line denotes direct link and the dashed line denotes relay link

value of energy stored at $R_{k}$ at the beginning of $i$-th signal block, and $O_{i}$ denotes the energy consumption for $i$-th signal block transmission. Thus, $O_{i}$ can be expressed as a stationary two-value random function, i.e.,

$$
P_{r}\left[O_{i}=M\right]=\left\{\begin{array}{rll}
\frac{P_{s d}^{\text {out }}}{K} & \text { if } \quad M=E_{r} \\
1-\frac{P_{s d}^{\text {out }}}{K} & \text { if } \quad M=0 \\
0 & \text { otherwise }
\end{array}\right.
$$

where factor $K$ represents the numbers of relay nodes who will be waked up with equal probability. Then, we get the expression of $S_{i+1}$ based on the aforementioned assumptions as

$$
S_{i+1}=\left\{\begin{array}{rll}
S_{i}+E_{i}-O_{i}, & \text { if } & S_{i}+E_{i}-O_{i} \in\left[0, E_{\max }\right] \\
S_{i}+E_{i}, & \text { if } & S_{i}+E_{i}-O_{i}<0 \\
E_{\max } & \text { if } & S_{i}+E_{i}-O_{i}>E_{\max }
\end{array}\right.
$$

Considering the possible energy consumption flow at $R_{k}$ from a statistical perspective, which is called as an onoff model and denoted by $S_{i}$. Namely, if $S_{i} \geq P_{r}, P_{r}$ can retransmit the signal successfully and the status of $S_{i}$ is defined as on,which is equivalent to the sum set of all the possible original statuses satisfying $S_{i} \mid S_{i} \geq P_{r}$. Otherwise, the status of $S_{i}$ is defined as off, so the transmission outage will occur. Therefore,

$$
P_{r}\left[\dot{S}_{i}=\text { on }\right]=\int_{P_{r}}^{E_{\max }} f_{s}(x) d x
$$

and

$$
P_{r}\left[S_{i}=o f f\right]=\int_{0}^{P_{r}} f_{s}(x) d x
$$

where $f_{s}(x)$ denotes the stationary probability distribution of $S_{i}$.
That is the relay-aided link will wake up if and only if the direct link is too bad, and the current available energy at the relay node is more than $E_{r}$, the energy-harvesting module at $R_{k}$ can be characterized by the parameter pair $\left(E_{r}, R_{k}\right)$, we can also say that the energy-harvesting module can provide an energy flow that is less than $E_{r}$ with the probability $p_{k}^{e x}$.

\section{Outage behavior analysis}

\subsection{Problem analysis}

Due to fluctuation of ambient renewable energy, the relay nodes cannot harvest enough energy to maintain working status. So, the outage event will occur.

According to aforementioned descriptions, that is to say, if the direct link channel is good, or the relay link's energy is more about $E_{r}$, and the relay link channel is good, the system can work well. We can express the overall successful communication events as

$$
\begin{aligned}
& \text { \{direct link }(S-D) \text { is good }\} \\
& \bigcup_{\kappa}\left\{\left\{\kappa \text { th relay link }\left(S-R_{k}-D\right) \text { is good }\right\}\right. \\
& \left.\bigcap\left\{\text { the energy at } R_{k} \text { is enough }\right\}\right\}
\end{aligned}
$$

Correspondingly, if the direct link channel is not good, at the same time, the relay link channel is not good, or the relay link node's energy is small than $E_{r}$, the system outage will occur. The overall outage events can be expressed as

$$
\text { \{direct link }(S-D) \text { is bad\} }
$$

$$
\begin{aligned}
& \bigcap_{\kappa}\left\{\left\{\kappa \text { th relay link }\left(S-R_{k}-D\right) \text { is bad }\right\}\right. \\
& \left.\bigcup\left\{\text { the energy at } R_{k} \text { is not enough }\right\}\right\}
\end{aligned}
$$


Assume the energy-harvesting modules owned by the relay nodes are independent from each other. The overall outage probability in (9) following the transmission protocol can be given by

$$
p_{\text {total }}^{\text {(out) }}=P_{s d}^{(\text {out })} \cdot \prod_{\kappa=1}^{K}\left[p_{k}^{e x}+\left(1-p_{k}^{e x}\right) \cdot P_{s r_{k} d}^{(\text {out })}\right]
$$

where $P_{s d}^{(\text {out })}$ denotes the outage probability of the direct link can not work, $P_{s r_{k} d}^{(\text {out }}$ denotes the outage probability of the relay link is not good or the energy of the relay node is small than $E_{r}$, and $p_{k}^{e x}$ is the probability of relay-aided link do not have enough energy to active itself to work, which have been discussed on the previous chapter.

In order to calculate $p_{\text {total }}^{\text {(out) }}$ we have a detail analysis about $P_{s d}^{\text {(out) }}$ and $P_{s r_{k} d}^{\text {(out) }}$ in the next step.

\subsection{Calculation of $P_{s d}^{\text {(out) }}$}

In direct transmission scenario, only the direct link between $S$ and $D$ is selected as a working link where all relay nodes do not work, and all the time slots in a signal block are used by the direct link.

The mutual information for the direction link of $S-D$ achieved by zero-mean circularly symmetric complex Gaussian input is defined as:

$I_{s d}=\log _{2}\left(1+P_{s} \frac{\left|h_{s d}\right|^{2}}{N_{D}}\right)=\log _{2}\left(1+\frac{P_{s}}{N_{D}} h_{0}\right)=\log _{2}\left(1+\gamma h_{0}\right)$ where $\gamma=\frac{P_{s}}{N_{D}}$ denotes SNR, and for $h_{0}=\left|h_{s d}\right|^{2}, h_{0}$ follows the Nakagami-m distribution, whose PDF and outage probability is expressed as respectively

$$
p\left(h_{0}\right)=\frac{m_{s d}^{m_{s d}} h_{0}^{m_{s d}-1}}{\Omega_{s d}^{m_{s d}} \Gamma\left(m_{s d}\right)} \exp \left(-\frac{m_{s d}}{\Omega_{s d}} h_{0}\right)
$$

Assume that the minimum acceptable rate equals to $R_{0}$, the outage probability $P_{s d}^{(\text {out })}\left[R_{s, d}<R_{0}\right]$ under direct transmission protocol can be expressed as

$$
\begin{aligned}
P_{s d}^{(\text {out })} & =P\left[\log _{2}\left(1+\gamma h_{0}\right) \leq R_{0}\right]=P\left[h_{0} \leq \gamma_{t h}^{s d}\right] \\
& =\int_{0}^{\gamma_{t h}^{s d}} \frac{m_{s d} m_{s d} h_{0}^{m_{s d}-1}}{\Omega_{s d}^{m_{s d}} \Gamma\left(m_{s d}\right)} \exp \left(-\frac{m_{s d}}{\Omega_{s d}} h_{0}\right) d h_{0} \\
& =\frac{m_{s d}}{\Omega_{s d} \Gamma\left(m_{s d}\right)} \int_{0}^{\gamma_{t h}^{s d}}\left(\frac{m_{s d}}{\Omega_{s d}} h_{0}\right)^{m_{s d}-1} \exp \left(-\frac{m_{s d}}{\Omega_{s d}} h_{0}\right) d h_{0} \\
& =\frac{1}{\Gamma\left(m_{s d}\right)} \int_{0}^{\frac{m_{s d}}{\Omega_{s d}} \gamma_{t h}^{s d}} t^{m_{s d}-1} e^{-t} d t, t=\frac{m_{s d}}{\Omega_{s d}} h_{0} \\
& =\frac{1}{\Gamma\left(m_{s d}\right)} \Gamma\left(m_{s d}, f r a c m_{s d} \Omega_{s d} \gamma_{t h}^{s d}\right) \\
& =1-\exp \left(-\frac{m_{s d}}{\Omega_{s d}} \gamma_{t h}^{s d}\right) \sum_{n=0}^{m_{s d}-1}\left(\frac{m_{s d} \gamma_{t h}^{s d}}{\Omega_{s d}}\right)^{n} \frac{1}{n !}
\end{aligned}
$$

where $\gamma_{t h}^{s d}=\frac{2^{R_{0}}-1}{\gamma}$

\subsection{Calculation of $P_{s r_{k} d}^{\text {(out) }}$}

For cooperative relay system [24, 25], if someone relay aided a link with enough energy among $K$ 's relay, it will be

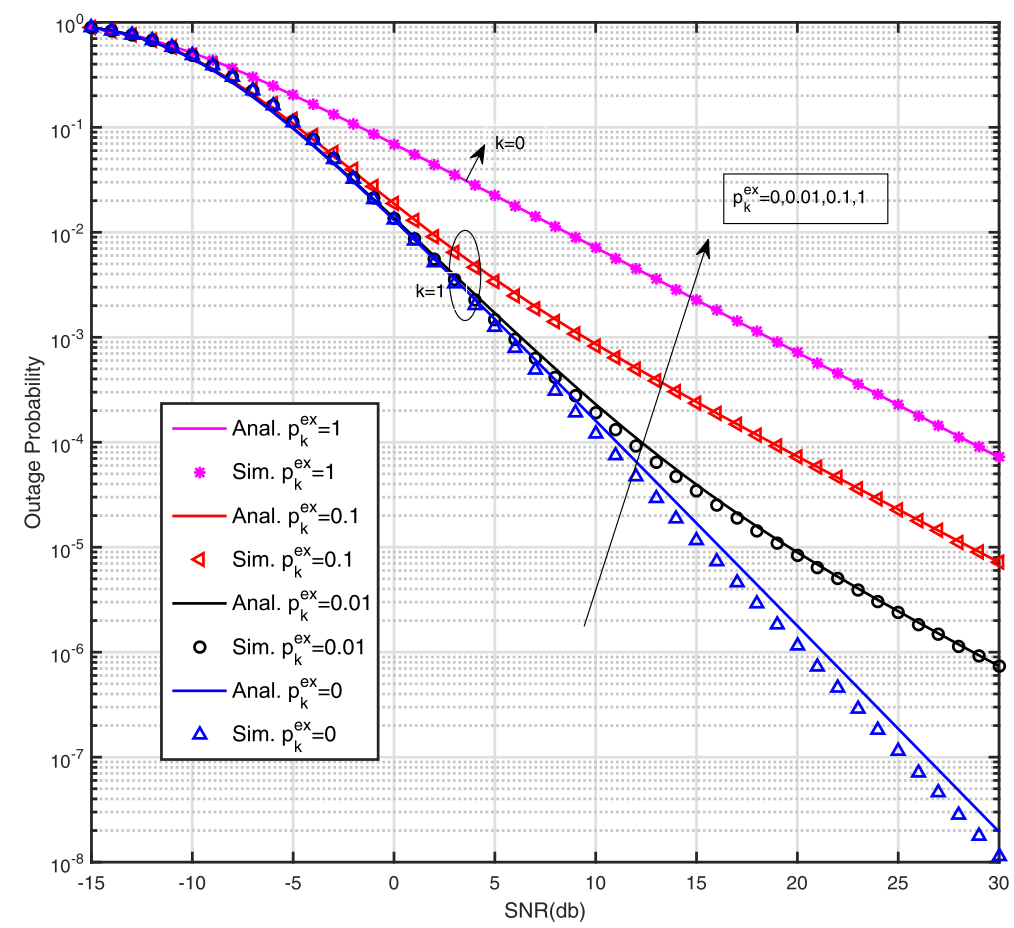

Fig. 2 Outage probability of daul hop energy-harvesting AF relay-aided systems with $m_{s d}=1, m_{s_{r} k}=1, m_{r_{k} d}=1, p_{1}^{e x}=\{0,0.01,0.1,1\}, k=1$ 
selected as a work node on the condition that the direct link cannot provide information transmission because of deep fading. The cooperative relay works as

Step 1: the system prefers to consider the direct link to transmit information with. If $\left|h_{s, d}\right|^{2}$ is bigger than the threshold value, the direct link shown in (1) will be selected to work for transmitting information. And the relay link will not need to be set up. Otherwise, go to Step 2.

Step 2: some relay link among K's, as shown in (2) and (3), will be selected and activated to work instead of a direct link. If it also fails, then there will be an outage event occurred.

Since the relay node $R_{k}$ is deployed to amplify-andforward relay scheme. Considering the relationship between received and signal $y_{s, r_{k}}(t)$ and retransmitted signal $x_{r_{k}}(t)$ at $R_{k}$, as shown in (2) and (3), we can get

$$
x_{r_{k}}(t)=G \cdot y_{s, r_{k}}(t)
$$

Correspondingly, the destination D's received instantaneous SNR [21] can be express as

$$
\begin{aligned}
\gamma_{e q} & =\frac{\left|h_{s r_{k}}\right|^{2}\left|h_{r_{k} d}\right|^{2} G^{2} P_{s}}{\left|h_{r_{k}}\right|^{2} G^{2} N_{R}+N_{D}}=\frac{\frac{\left|h_{s r_{k}}\right|^{2} P_{s}}{N_{R}} \frac{\left|h_{r_{k} d}\right|^{2} P_{r_{k}}}{N_{D}}}{\frac{\left|h_{s r_{k}}\right|^{2} P_{s}}{N_{R}}+\frac{\left|h_{r_{k}}\right|^{2} P_{r_{k}}}{N_{D}}+1} \\
& =\frac{\gamma_{s r_{k}} \gamma_{r_{k}} d}{\gamma_{s r_{k}}+\gamma_{r_{k}} d+1} \\
G^{2} & =\frac{P_{r_{k}}}{\left|h_{s r_{k}}\right|^{2} P_{s}+N_{R}}
\end{aligned}
$$

Then we can get the mutual information for the $S-R_{k}-D$ link as

$$
I_{s r_{k} d}=\frac{1}{2} W \log _{2}\left(1+\gamma_{e q}\right)
$$

Given $\gamma_{e q}$ in (15), which is the random variable denoting the received SNR at destination node $D$, then we can derive exact expressions for the CDF of $\gamma_{e q}$. And the CCDF of $\gamma_{(e q)}$ is $\bar{F}_{\gamma_{e q}}(\gamma)[26,27]$

$$
\left.\begin{array}{rl}
\bar{F}_{\gamma_{e q}}(\gamma)= & 2 \exp \left[-\left(\frac{m_{s r_{k}}}{\Omega_{s r_{k}}}+\frac{m_{r_{k} d}}{\Omega_{r_{k}} d}\right) \gamma\right] \\
& \cdot \sum_{n=0}^{m_{s r_{k}}-1} \sum_{k=0}^{m_{r_{k}} d^{-1}} \sum_{m=0}^{k}\left\{\frac{\frac{\Omega_{s r_{k}}}{m_{s r_{k}}}}{m !(k-m) ! n !\left(m_{s r_{k}}-n-1\right) !}\right. \\
& K_{n-m+1}\left(2 \sqrt{\frac{m_{s r_{k}} m_{r_{k}} d \gamma(\gamma+1)}{\Omega_{s r_{k}} \Omega_{r_{k}} d}}\right)\left(1+\frac{1}{\gamma}\right)^{\frac{n+m+1}{2}} \gamma^{m_{s r_{k}}+k}
\end{array}\right\}
$$

So, CDF of $\gamma_{(e q)}$ is $F_{\gamma_{e q}}(\gamma)$, which is given by

$$
P_{s r_{k} d}^{\text {(out) }}=P_{r}\left(\gamma_{e q}<\gamma_{t h}\right)=F_{\gamma_{e q}}(\gamma)=1-\bar{F}_{\gamma_{e q}}(\gamma)
$$

\subsection{System overall outage probability}

Substituting (13) and (19) into (10), the explicit closedform expression of outage probability for the relayaided cooperative transmission protocol by the harvested energy can be explained as follows

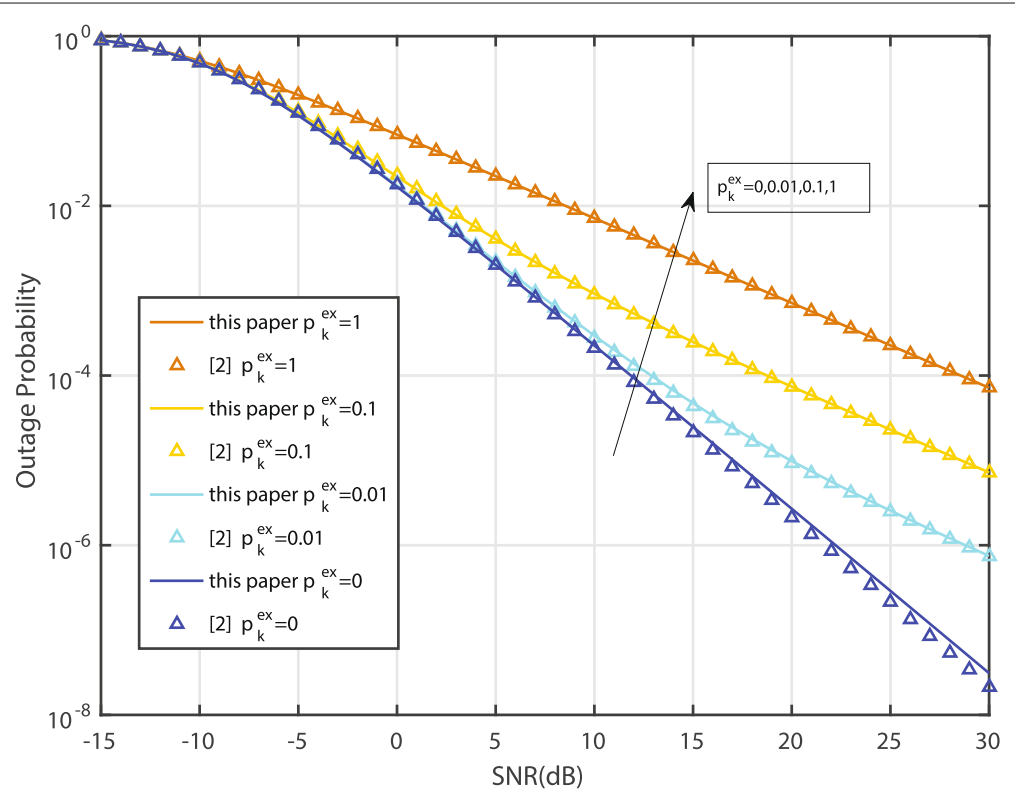

Fig. 3 Outage probability of dual hop energy-harvesting AF relay-aided systems compare with [2] at $m_{s d}=1, m_{s, k}=1, m_{r_{k} d}=1$, $p_{1}^{e x}=\{0,0.01,0.1,1\}, k=1$ 


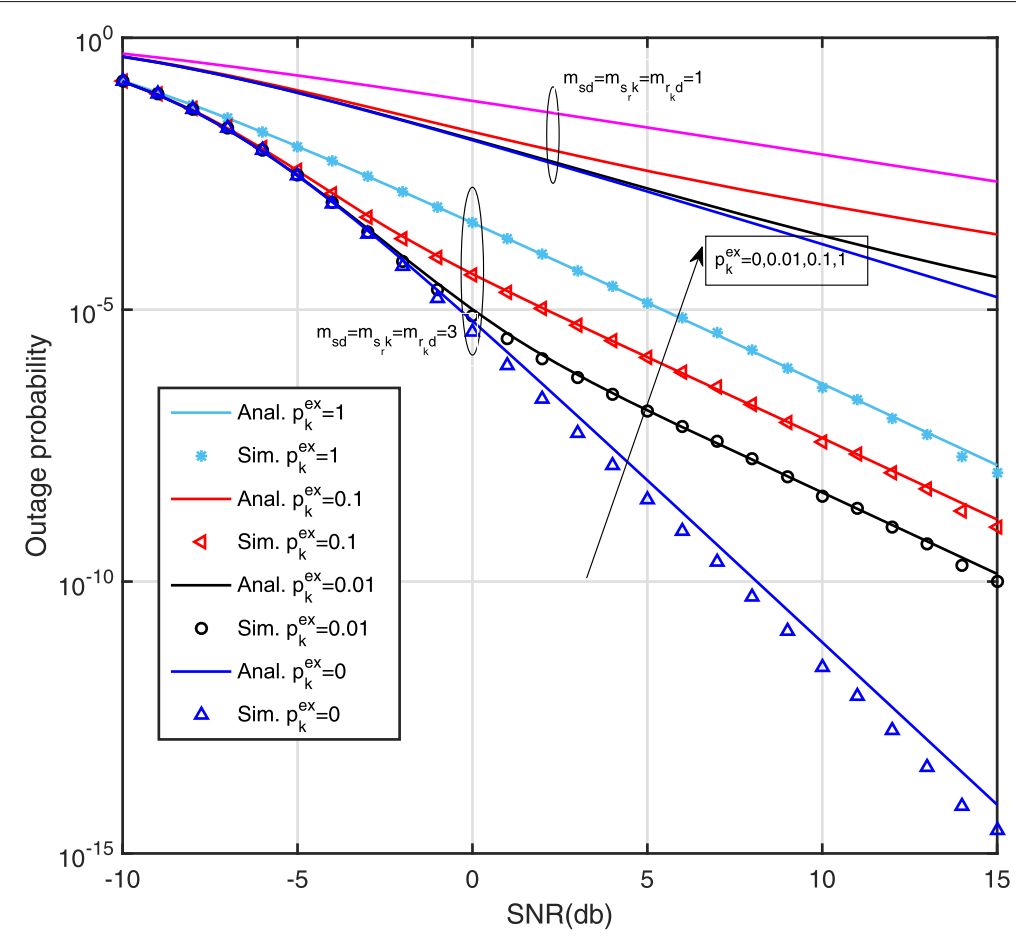

Fig. 4 Outage probability of dual hop energy-harvesting AF relay-aided systems with $m_{s d}=3, m_{s_{r} k}=3, m_{r_{k} d}=3, p_{1}^{e x}=\{0,0.01,0.1,1\}$, and $k=1$

$$
\begin{aligned}
& P_{\text {total }}^{(\text {out })}=\left\{1-\exp \left(-\frac{m_{s d}}{\Omega_{s d}} \gamma_{t h}^{s d}\right) \sum_{n=0}^{m_{s d}-1}\left(\frac{m_{s d} \gamma_{t h}^{s d}}{\Omega_{s d} n !}\right)^{n}\right\} \\
& \text {. } \prod_{k=1}^{K}\left\{p_{k}^{e x}+\left(1-p_{k}^{e x}\right)\left\{1-2 \exp \left[-\left(\frac{m_{s r_{k}}}{\Omega_{s r_{k}}}+\frac{m_{r_{k} d}}{\Omega_{r_{k} d}}\right) \gamma\right]\right.\right.
\end{aligned}
$$

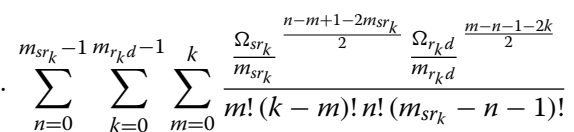

$$
\begin{aligned}
& \left.\left.\cdot K_{n-m+1}\left(2 \sqrt{\frac{m_{s r_{k}} m_{r_{k}} d \gamma(\gamma+1)}{\Omega_{s r_{k}} \Omega_{r_{k}} d}}\right)\left(1+\frac{1}{\gamma}\right)^{\frac{n+m+1}{2}} \gamma^{m_{s r_{k}}+k}\right\}\right\}
\end{aligned}
$$

\section{Simulation results}

This section studies how the outage probability changes with different parameters. It is assumed that the available frequency bandwidth for the whole network is $W=2 \mathrm{Mhz}$, and the minimum acceptable rate $R_{0}=200 \mathrm{kbps}, P_{s}=P_{r}=$ $P_{0}=1 w$. Monte Carlo simulations were carried out by $2 \times$ $10^{8}$ sample points with intel(R) Core(TM) i7-6700HQ, 4 cores CPU@2.60 GHz and 16.0 GB RAM.

In Fig. $2, K=1$. It shows the outage probability of cooperative networks as a function of $\operatorname{SNR}$ (i.e., $S N R=P_{0} / \sigma_{0}^{2}$ ) when the energy-exhausted probability $P_{1}^{e x}$ at $R$ is $1,0.1$, 0.01 , and 0 with $m_{s d}=1, m_{s_{r} k}=1$, and $m_{r_{k} d}=1$. Obviously, when $P_{1}^{e x}=1$, it is equivalent to the traditional system with direct transmission protocol. In addition, when $P_{1}^{e x}=0$, it is equivalent to the system in which the relay node is powered by a constant power system. At last, we can observe in Fig. 2 that the energy-harvesting relay node can improve the outage performance of the system significantly compared with traditional direct transmission protocol, which has no relay link.

In Fig. $3, K=1$. It shows the outage probability of cooperative networks as a function of $\operatorname{SNR}\left(\right.$ i.e., $\left.S N R=P_{0} / \sigma_{0}^{2}\right)$ when the energy-exhausted probability $P_{1}^{e x}$ at $\mathrm{R}$ is $1,0.1$, 0.01 and 0 , respectively with $m_{s d}=1, m_{s_{r} k}=1, m_{r_{k} d}=1$, it is coordinated to the curve of the work [11] at the Rayleigh channels parameters.

In Fig. $4, K=1$. It shows the outage probability of cooperative networks as a function of $\operatorname{SNR}\left(\right.$ i.e., $\left.S N R=P_{0} / \sigma_{0}^{2}\right)$ when the energy-exhausted probability $P_{1}^{e x}$ at $R$ is $1,0.1$, 0.01 , and 0 , with $m_{s d}=3, m_{s_{r} k}=3, m_{r_{k} d}=3$, Similarly, when $P_{1}^{e x}=1$, it is equivalent to the traditional system with direct transmission protocol. In addition, when $P_{1}^{e x}=0$, it is equivalent to the system in which the relay node is powered by constant power system. At last, we can observe in Fig. 4 that the energyharvesting relay node can improve the outage performance of the system significantly compared with traditional direct transmission protocol during the concerned SNR range. But comparing with the results from Fig. 2, we can find that the downward trend is more fast with the increasing of SNR. That is to say, with the increment 


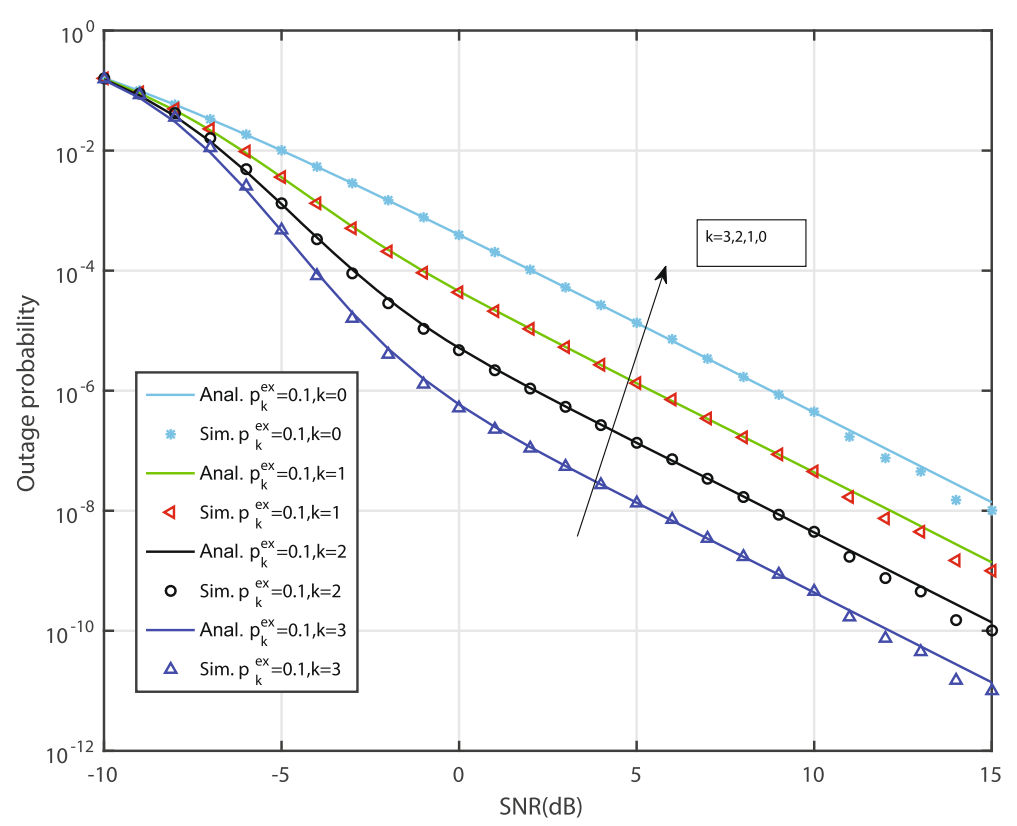

Fig. 5 Outage probability of dual hop energy-harvesting AF relay-aided systems with $m_{s d}=3, m_{s_{r} k}=3, m_{r_{k} d}=3,\left(p_{1}^{e x}, p_{2}^{e x} \ldots p_{N}^{e x}\right)=0.1$, and $k=\{3,2,1,0\}$

of relay link, we can get better performance comparing with the scenario in the Fig. 2, which has one relay link only.

In Fig. 5, it shows the outage probability of cooperative networks as a function of SNR when the number of available relay nodes $K$ is $0,1,2,3$, respectively with $m_{s d}=3$, $m_{s_{r} k}=3, m_{r_{k} d}=3$, and $\left(P_{1}^{e x}, P_{2}^{e x} \ldots P_{N}^{e x}\right)=0.1$. It can be observed that the system performance can be significantly improved with the increment of the number of available relay nodes $K$.

In Fig. 6, it shows the outage probability of cooperative networks as a function of $K$ when the SNR is 15,10 , and $5 \mathrm{~dB}$, respectively with $m_{s d}=3, m_{s_{r} k}=3, m_{r_{k} d}=3$, and $\left(P_{1}^{e x}, P_{2}^{e x} \ldots P_{N}^{e x}\right)=(0.1,0.1 \ldots, 0.1)$. It can be observed that the system performance can improved with the linear increment of the SNR. In Table 1, it shows that the

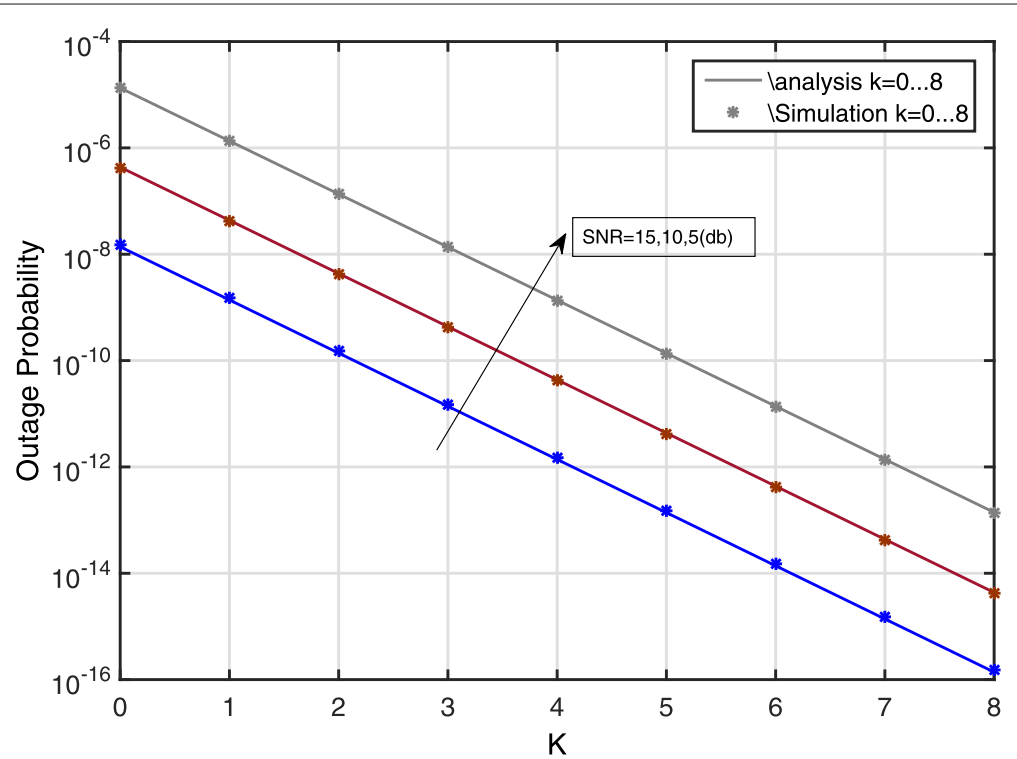

Fig. 6 Outage probability of dual hop energy-harvesting AF relay-aided systems with $m_{s d}=3, m_{s_{r} k}=3, m_{r_{k} d}=3,\left(p_{1}^{e x}, p_{2}^{e x} \ldots p_{N}^{e x}\right)=0.1$, snr $=\{15,10,5\}$, and $k=\{0,1,2,3,4,5,6,7,8\}$ 
Table 1 Operation time(s) of our numerical calculate and Monte Carlo method

\begin{tabular}{lllll}
\hline & Fig. 2 & Fig. 4 & Fig. 5 & Fig. 6 \\
\hline Our numerical calculate & 0.131 & 0.937 & 0.209 & 2.084 \\
Monte Carlo method & 1024.163 & 1024.163 & 634.919 & 123.015 \\
\hline
\end{tabular}

numerical calculate time is more less than the Monte Carlo time, the theoretical results can be applied without running the complex Monte Carlo simulation in the practical application.

\section{Conclusions}

In this paper, we presented the performance of cooperative networks aided by an on-off Markov model energyharvesting relay node in Nakagami-m fading scenario. With the help of some approximations, we got the explicit closed-form expression of outage probability of cooperative system based on the nonidentical Nakagami-m fading channels. The result of Monte Carlo method correlated with numerical analytical results reported by previous studies. Via simulations, it was shown that the system outage performance have been improved when natural source $\mathrm{EH}$ relays were employed. Besides, the more relays, the better system performance.

\section{Acknowledgements}

This work was supported by the National Science Foundation of China(NSFC), no. 61672217,61304208 , and also by the Foundation of the Science and technology project of Hunan Provincial Department of Education, no. 15C1414.

\section{Funding}

This paper was supported by the National Natural Science Foundation general projects, China (No. 61672217), by the National Natural Science Foundation general projects, China (No. 61304208), and also by the Foundation of the Science and technology project of Hunan Provincial Department of Education (No. 15C1414).

\section{Authors' contributions}

SZ has fulfilled all the system modeling, analysis, simulation, and drafting the article. RL has helped revise the manuscript. HH has given critical revision of the article and has helped revise the manuscript. All authors read and approved the final manuscript.

\section{Competing interests}

The authors declare that they have no competing interests.

\section{Publisher's Note}

Springer Nature remains neutral with regard to jurisdictional claims in published maps and institutional affiliations.

\footnotetext{
Author details

${ }^{1}$ College of Comuputer Science and Electronic Engineering, and the Key Laboratory for Embedded and Network Computing, Hunan University, Changsha, People's Republic of China. ${ }^{2}$ School of Computer and Information Engineering, Central South University of Forest and Technology, Changsha, People's Republic of China.
}

Received: 4 August 2017 Accepted: 6 December 2017

Published online: 16 March 2018

\section{References}

1. R Jiang, K Xiong, P Fan, Y Zhang, Z Zhong, Optimal design of SWIPT systems with multiple heterogeneous users under non-linear energy harvesting model. IEEE Access. 5, 11479-11489 (2017)

2. X Di, KXiong, P Fan, HC Yang, Simultaneous wireless information and power transfer in cooperative relay networks with rateless codes. IEEE Trans. Veh. Technol. 66(4), 2981-2996 (2017)

3. K Janghel, S Prakriya, Performance of secondary network with primary beamforming-assisted energy harvesting transmitters. PP(99), 1-1 (2017)

4. KXiong, P Fan, C Zhang, KB Letaief, Wireless information and energy transfer for two-hop non-regenerative MIMO-OFDM relay networks. IEEE J. Sel. Areas Commun. 33(8), 1595-1611 (2015)

5. NL Quang, TD Nhu, QB Vo Nguyen, An Beongku, Full-duplex distributed switch-and-stay networks with wireless energy harvesting: design and outage analysis. EURASIP J. Wirel. Commun. Netw. 2016 (2016)

6. KXiong, P Fan, T Li, KB Letaief, Group cooperation with optimal resource allocation in wireless powered communication networks. IEEE Trans. Wirel. Commun. 16(6), 3840-3853 (2017)

7. WK Xu, Z Yang, ZG Ding, L Wang, PZ Fan, Wireless information and power transfer in two-way relaying network with non-coherent differential modulation. EURASIP J. Wirel. Commun. Netw. 2015 (2015)

8. KXiong, P Fan, Y Lu, KB Letaief, Energy efficiency with proportional rate fairness in multirelay OFDM networks. IEEE J. Sel. Areas Commun. 34(5), 1431-1447 (2016)

9. KXiong, P Fan, HC Yang, KB Letaief, Space-time network coding with overhearing relays. IEEE Trans. Wirel. Commun. 13(7), 3567-3582 (2014)

10. S Luo, R Zhang, TJ Lim, Optimal save-then-transmit protocol for energy harvesting wireless transmitters. IEEE Trans. Wirel. Commun. 12(3), 1196-1207 (2013)

11. T Li, P Fan, KB Letaief, Outage probability of energy harvesting relay-aided cooperative networks over Rayleigh fading channel. IEEE Trans. Vehicular Technol. 65(2), 972-978 (2016)

12. AA Nasir, X Zhou, S Durrani, RA Kennedy, Relaying protocols for wireless energy harvesting and information processing. IEEE Trans. Wirel. Commun. 12(7), 3622-3636 (2013)

13. J Liu, KXiong, $\mathrm{P}$ Fan, Z Zhong, RF energy harvesting wireless powered sensor networks for smart cities. IEEE Access. 5, 9348-9358 (2017)

14. KXiong, P Fan, T Li, KB Letaief, Rate-energy region of SWIPT for MIMO broadcasting under nonlinear energy harvesting model. IEEE Trans. Wirel. Commun. 99, 1965-1970 (2017)

15. KXiong, P Fan, T Li, KB Letaief, Outage probability of space-time network coding over Rayleigh fading channels. IEEE Trans. Veh. Technol. 63(4), 1965-1970 (2014)

16. R Jiang, K Xiong, P Fan, Y Zhang, Z Zhong, Outage analysis and optimization of SWIPT in network-coded two-way relay networks. Mobile Inf. Syst (2017)

17. A Bletsas, H Shin, MZ Win, Cooperative communications with outage-optimal opportunistic relaying. IEEE Trans. Wirel. Commun. 6(9), 3450-3460 (2007)

18. N Kapucu, M Bilim, I Develi, Outage probability analysis of dual-hop decode-and-forward relaying over mixed Rayleigh and generalized gamma fading channels. Wirel. Pers. Commun. 71(2), 947 (2013)

19. P Kumar, S Majhi, Y Nasser, in Proc.Communications and Informatics (ICACCI) 2016 Int. Conf. Advances in Computing. Analysis of outage performance of opportunistic af OFDM relaying in Nakagami-m channels, (2016), pp. 2527-2531

20. SL Wang, TM Wu, in Proc.IEEE 80th Vehicular Technology Conf. (VTC2014-Fall). Performance analyses of two-way AF relaying over nakagami-m frequency-selective fading channels, (2014), pp. 1-5

21. D Senaratne, C Tellambura, Unified exact performance analysis of two-hop amplify-and-forward relaying in Nakagami fading. IEEE Trans. Veh. Technol. 59(3), 1529-1534 (2010)

22. Y Zhang, K Xiong, P Fan, HC Yang, X Zhou, Space-time network coding with multiple AF relays over Nakagami-m fading channels. IEEE Trans. Veh. Technol. 66(7), 6026-6036 (2017)

23. KXiong, P Fan, Z Xu, HC Yang, KB Letaief, Optimal cooperative beamforming design for MIMO decode-and-forward relay channels. IEEE Trans. Signal Process. 62(6), 1476-61489 (2014)

24. JN Laneman, DNC Tse, GW Wornell, Cooperative diversity in wireless networks: efficient protocols and outage behavior. IEEE Trans. Inf. Theory. 50(12), 3062-3080 (2004) 
25. MO Hasna, MS Alouini, A performance study of dual-hop transmissions with fixed gain relays. IEEE Trans. Wirel. Commun. 3(6), 1963-1968 (2004)

26. IS Gradshteyn, IM Ryzhik, Tables of Integrals, Series, and Products, 7th, (2007)

27. AF Pun, C Vldeanu, in Proc.10th Int. Conf. Communications (COMM). On the outage probability of dual-hop AF relaying over nakagami-m fading channels, (2014), pp. 1-4

\section{Submit your manuscript to a SpringerOpen ${ }^{\odot}$ journal and benefit from:}

- Convenient online submission

- Rigorous peer review

- Open access: articles freely available online

- High visibility within the field

- Retaining the copyright to your article

Submit your next manuscript at $\gg$ springeropen.com 